\title{
A New Perspective on the Role of A-site Cation in Perovskite Solar Cells
}

\author{
Chang Woo Myung, ${ }^{1}$ Jeonghun Yun, ${ }^{1}$ Geunsik Lee, ${ }^{2}$ and Kwang S. Kim ${ }^{1}$ \\ ${ }^{1}$ Center for Superfunctional Materials, Department of Chemistry and Department of Physics, \\ Ulsan National Institute of Science and Technology (UNIST), Ulsan 44919, Korea \\ ${ }^{2}$ Department of Chemistry and Department of Physics, \\ Ulsan National Institute of Science and Technology (UNIST), Ulsan 44919, Korea
}

(Dated: November 7, 2018)

\begin{abstract}
As the race towards higher efficiency for inorganic/organic hybrid perovskite solar cells (PSCs) is becoming highly competitive, a design scheme to maximize carrier transport towards higher power efficiency has been urgently demanded. Here, we unravel a hidden role of A-site cation of PSCs in carrier transport which has been largely neglected, i.e., tuning the Frhlich electron-phonon (e-ph) coupling of longitudinal optical (LO) phonon by A-site cations. The key for steering Frhlich polaron is to control the interaction strength and the number of proton (or lithium) coordination to halide ion. The coordination to $\mathrm{I}^{-}$alleviates electron-phonon scattering by either decreasing the Born effective charge or absorbing the LO motion of I. This novel principle discloses lower electron-phonon coupling by several promising organic cations including hydroxyl-ammonium cation $\left(\mathrm{NH}_{3} \mathrm{OH}^{+}\right)$ and possibly $\mathrm{Li}^{+}$solvating methylamine $\left(\mathrm{Li}^{+} \ldots \mathrm{NH}_{2} \mathrm{CH}_{3}\right)$ than methyl-ammonium cation. A new perspective on the role of A-site cation could help in improving power efficiency and accelerating the application of PSCs.
\end{abstract}

Solar energy is a highly efficient and eco-friendly source for future energy harvesting. In particular, inorganic/organic PSCs of ABX3 $(\mathrm{A}=\mathrm{Cs}+, \mathrm{CH} 3 \mathrm{NH} 3+$, etc.; $\mathrm{B}=\mathrm{Pb} 2+; \mathrm{X}=\mathrm{Cl}-, \mathrm{Br}-$ or I-) show extraordinary solar cell efficiencies exceeding $22 \%$ [1] with unusual characteristics, 2, 4, which attracts tremendous attention as most promising large-scale solar energy conversion materials. 5] One key origin of high efficiency arises from high carrier mobility $(\mu) 10^{1}-10^{3}\left(\mathrm{~cm}^{2} \mathrm{~V}^{-1} \mathrm{~s}^{-1}\right)[6$ 8 even in the presence of defects. 9] While the carrier transport exhibits remarkable features in experiments, there is still a gap of understanding. One aspect of this difficulty stems from significant electron-phonon (e-ph) interactions, [2, 8, 10, 13, which complicates band pictures. Another aspect is due to the A-site cation that rotates 14 or even diffuses across the material, causing I-V hysteresis. 15 Although recent discovery of various types of cations has greatly advanced the efficiency of PSCs, [16] it also has brought about more ambiguity on the role of cations.

In general, electrons in polar crystal experience the deformation potential in addition to Bloch potential due to large polarity of ionic bonding. The charge carriers are then described by polaron quasiparticles originating from coupling between electrons and phonons. For PSCs, there has been a critical debate on the source of e-ph coupling, acoustic vs. longitudinal. From the temperature dependence of $\mu \sim T^{-3 / 2}$ around room temperature, acoustic phonons have been considered as the main source of e-ph coupling. [11, 17] However, a theoretical study of acoustic phonon scattering predicts 18 $\mu$ to be several $10^{3} \mathrm{~cm}^{2} V^{-1} \mathrm{~s}^{-1}$, far above the experimental values. Meanwhile, recent studies suggest LO phonons as the main source of polaron states. [19, 20 The working temperature of cubic CH3NH3PbI3 $(330 \mathrm{~K}) 21$ is well above the Debye temperature $\Theta_{D} \sim 175 \mathrm{~K}[22$ which is a representative excitation energy scale of the highest phonon mode. Therefore, it is plausible that at working temperatures of PSC, high energy PbI3 LO phonons are largely populated.

In this study, we unveil a hidden role of A-site cation in polaron picture of PSCs by accounting for the e-ph interactions regarding both A-site cation and LO phonon scattering at the first principles level.[18, 19 We used the Frohlich polaron model[23] which is suitable for large bandwidth $(W)$ limit $W \gg \hbar \omega_{p h}$ by considering Frohlich vertex. 24 We cast light on the role of various organic cations in polaronic carrier transport and propose a design principle of choosing A-site cation towards higher carrier mobility of PSCs, which is very crucial for photocreated carriers to travel to collector in solar cells. The phonon dispersion, spectral function, and carrier lifetime of lead iodide $\left(\mathrm{APbI}_{3}\right)$ perovskite are calculated for prospective $\mathrm{A}$-site cations including existing cations $\mathrm{A}=$ $\mathrm{Cs}^{+}, \mathrm{CH}_{3} \mathrm{NH}_{3}^{+}$(methyl-ammonium cation; $\mathrm{MA}^{+}$) based on density functional theory (DFT), density functional perturbation theory (DFPT), and many-body theory. 24] We find a novel design principle for A-site cation and uncover some of unexplored A-site cations showing better charge transport property.

There has been a little understanding regarding the role of A-site cation particularly associated with polaron nature of the material. It is important for the size of A-site cation to be suitable for a semi-empirical factor called the Goldschmidt tolerance factor $t=\left(r_{A}+\right.$ $\left.r_{I}\right) / \sqrt{2}\left(r_{P b}+r_{I}\right),[25]$ where its ionic radius of A-site cation $r_{A}=r_{c m}+r_{i o n}$ with $r_{c m}$ being a distance between the center of mass and the farthest atom and $r_{i o n}$ being the corresponding ionic radius except hydrogen. 26] Among many candidates, inorganic cations are not appropriate according to the Goldschmidt factor because of its small ionic radius, $t<1$. The size of A-site cation also controls a degree of rotation of octahedral, thereby tuning the bandgap of PSCs. 27] One of origins of the structural 

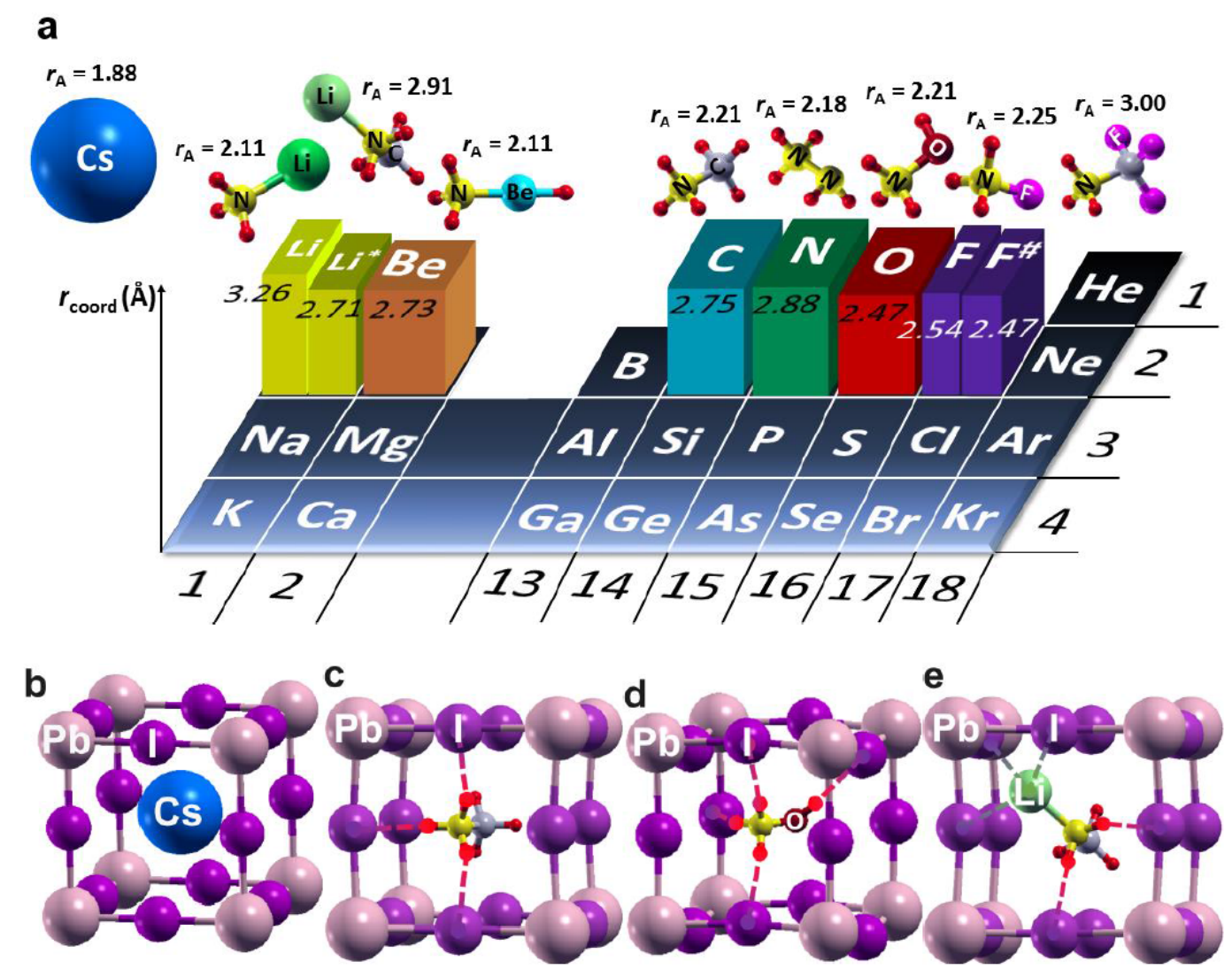

FIG. 1. a) Average values of coordinating distances rcoord to $\mathrm{I}^{-}$in cubic lead iodide PSCs for various A-site organic cations and estimated ionic radii $r_{A}$ of A-site cations. $\mathrm{Li}^{*}$ refers to $\mathrm{LiNH}_{2} \mathrm{CH}_{3}^{+}$and $\mathrm{F}^{\#}$ refers to $\mathrm{NH}_{3} \mathrm{CF}_{3}^{+} \cdot \mathrm{NH}_{3} \mathrm{BH}_{2}^{+}$does not form

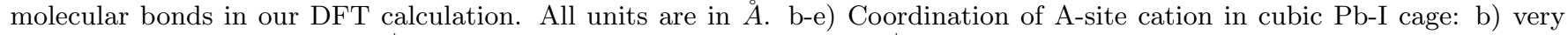
weakly dodeca-coordinated $\mathrm{Cs}^{+}$by $\mathrm{I}^{-}$, c) strongly tri-coordinated $\mathrm{MA}^{+}$by three $\mathrm{I}^{-} \ldots \mathrm{HN}$ hydrogen bonds, d) strongly tetracoordinated $\mathrm{NH}_{3} \mathrm{OH}^{+}\left(\mathrm{HA}^{+}\right)$by three $\mathrm{I}^{-} \ldots \mathrm{HN}$ and one $\mathrm{I}^{-} \ldots \mathrm{HO}$ hydrogen bonds, e) strongly penta-coordinated $\mathrm{LiNH}_{2} \mathrm{CH}_{3}^{+}$ $\left(\mathrm{LM}^{+}\right)$by two $\mathrm{I}^{-} \ldots \mathrm{HN}$ hydrogen bonds and three $\mathrm{I}^{-} \ldots \mathrm{Li}$ ionic bonds.

instability of most-widely used $\mathrm{MAPbI}_{3}$ is from its small $r_{A}$ being $t \sim 0.9<1.0$. One way to circumvent this problem is to tune the size of A-site cation (or halides), and many researchers tried to increase the number of carbon chain or amine group of MA+. [26, 28, As the low energy spectra near the Fermi level $\left(E_{F}\right)$ contain only $\mathrm{Pb}^{2+}$ (conduction) and $\mathrm{I}^{-}$(valence) states without $\mathrm{A}$ site cation, the role of A-site cations has been overlooked with a limited understanding as a structural tuner with its ionic radius.

For polar semiconductors, strong ionic bonding forms a dipole $p \sim e \omega_{q}^{1 / 2} Z_{\kappa}^{*} e(q) e^{i q R}$ within a unit cell, where $e$ is electron charge, $\omega_{q}$ is phonon frequency, $Z_{\kappa}^{*}$ is the Born effective charge which is a measure of polarization or dipole per unit cell, and $e(q)$ is eigenmode. Because the dipole-dipole interaction decays as $R_{p}^{-2}$, the interaction in momentum space diverges as $q^{-1}$ for long range components of phonon $q \rightarrow 0$. 29. Only the LO mode in long range limit provides a major contribution to phonon coupling to band edge states. Ab initio coupling matrix element of this long range part of phonon mode $\nu$ and bands $\mathrm{n}, \mathrm{m}$ at wave vector $q$ is given by 24

$$
g_{n m}(k, \omega)=i \frac{4 \pi}{\Omega} \frac{e^{2}}{4 \pi \epsilon_{0}} \sum_{\kappa, G \neq-q}\left(\frac{\hbar}{2 N M_{\kappa} \omega_{q \nu}}\right)^{1 / 2} \frac{(q+G) Z_{\kappa}^{*} e_{\kappa}(q)}{(q+G) \epsilon_{\infty}(q+G)}\left\langle\psi_{m k+q}\left|e^{i(q+G)\left(r-\tau_{\kappa}\right)}\right| \psi_{n k}\right\rangle
$$

where $\Omega$ is the unit cell volume, $N$ the number of unit cells in the supercell, $\kappa$ atom index within a unit cell, $\epsilon_{0}$ the vacuum permittivity, $\hbar$ the reduced Planck constant, $\left|\psi_{n k}\right\rangle$ and $\left|\psi_{m k+q}\right\rangle$ are initial and final electronic states. In order to reduce e-ph interactions that hinder carrier transport in semiconductor, we need to decrease the dipole of a given unit cell $(p)$, i.e., Born effective charge $Z_{\kappa}^{*}$ and vibrational eigenmode component $e(q)$. 
From a given observation in Equation (1), we note a novel design principle of A-site cation towards high efficiency of PSCs. A simple rule is to let the A site cation be nearer to I and to increase the number of A site cations coordinating to I. We expect that the wellchosen cations are able to suppress e-ph coupling effectively by decreasing the $Z_{\kappa}^{*}$ of $\mathrm{Pb}$ and I with decreased distance from the A cation to I and by reducing of $\mathrm{Pb} 2+$ and I- ions with increased coordination number of organic cations. A recently measured reorientation time scale for the MA+ molecule in a pseudo-cubic cage $(\sim 14 p s)$ is long enough to participate in lattice vibrations of $\mathrm{Pb}-$ I frame to form the polaron state.14 Stemming from the widely used $\mathrm{NH}_{3} \mathrm{CH}_{3}{ }^{+}\left(\mathrm{MA}^{+}\right)$, we have substituted $\mathrm{CH}_{3}$ by $\mathrm{Li}\left(\mathrm{NH}_{3} \mathrm{Li}^{+}\right), \mathrm{BeH}, \mathrm{BH}_{2}, \mathrm{NH}_{2}\left(\mathrm{NH}_{3} \mathrm{NH}_{2}{ }^{+}\right.$: hydrazinium: $\left.\mathrm{HZ}^{+}\right), \mathrm{OH}\left(\mathrm{NH}_{3} \mathrm{OH}^{+}\right.$: hydroxyl-ammonium cation: $\left.\mathrm{HA}^{+}\right), \mathrm{F}\left(\mathrm{NH}_{3} \mathrm{~F}^{+}\right.$: fluoro-ammonium cation, which is denoted as $\mathrm{fA}^{+}$in consideration that $\mathrm{FA}^{+}$is ofent used to denote formamidinium cation) and calculated their average coordination distance and ionic radius. While $\mathrm{HZ}^{+}$and $\mathrm{HA}^{+} 30$, 31 are available in laboratories, $\mathrm{fA}^{+}$is difficult to be synthesized, though its spectra were observed from experiments. 32 However, to understand the role of cations in the A-site, we investigated the series of cations mentioned above. The $\mathrm{HZ}^{+}$ results are found to be very similar to $\mathrm{MA}^{+}$, so the discussion of $\mathrm{HZ}^{+}$is omitted here to reduce the complexity in comparison, and we will focus on the comparison of $\mathrm{Cs}^{+}$with $\mathrm{MA}^{+}, \mathrm{HA}^{+}$and $\mathrm{fA}^{+}$. Using DFT calculations, we find that the average coordination distance rcoord for $\mathrm{MA}^{+}(2.75 \AA)$ decreases to $2.47 \AA$ for $\mathrm{HA}^{+}$and 2.54 $\AA$ for $\mathrm{fA}^{+}$(Figure 1a). These shortened ionic hydrogen bonds between $\mathrm{I}^{-}$and $\mathrm{H}\left(\mathrm{NH}_{2}\right)$ in $\mathrm{HA}^{+} / \mathrm{fA}^{+}$(decreased by $0.2-0.3 \AA$ with respect to $\mathrm{C}$ ) give smaller ( $\mathrm{Pb}$, I) than that in $\mathrm{MA}^{+}$. This suggests better performance by A-site cations with $\mathrm{OH}$ or $\mathrm{F}$ than $\mathrm{CH}_{3}$. Another way to achieve our goal is fluorinating $\mathrm{H}$ atoms of $\mathrm{CH}_{3}$ to form $\mathrm{NH}_{3} \mathrm{CF}_{3}{ }^{+}$(tri-fluorinated methyl ammonium: $\mathrm{TA}^{+}$) or substituting $\mathrm{H}-\mathrm{N}$ to $\mathrm{Li}-\mathrm{N}$ to form $\mathrm{LiNH}_{2} \mathrm{CH}_{3}{ }^{+}$ (lithium-methylamine: $\mathrm{LM}^{+}$). $\mathrm{LM}^{+}$is again difficult to be synthesized, though its spectra were observed from experiments. 33. This is however also investigated to understand what kind of molecular type is useful to reduce the e-ph coupling.

Various types of configurations of A-site cations with surrounding $\mathrm{I}^{-}$ions are observed using DFT geometry optimization (Figures $1 \mathrm{~b}$ to $1 \mathrm{e}$ ). Inorganic $\mathrm{Cs}^{+}$is very weakly dodeca-coordinated by $\mathrm{I}^{-}$with rather large distances $\left(4.5 \AA\right.$ ). A typical A-site cation $\mathrm{MA}^{+}$forms three strong ionic hydrogen bonds between $\mathrm{I}^{-}$and $\mathrm{H}$, namely tri-coordination (Figure 1c). $\mathrm{HA}^{+}$forms additional coordination employed by $\mathrm{OH}$ and $\mathrm{I}^{-}$(Figure 1d). Moreover, $\mathrm{LM}^{+}$having $\mathrm{Li}^{+}$instead of proton can attach noncovalently to five $\mathrm{I}^{-} \mathrm{s}$ as two $\mathrm{I}^{-} \mathrm{H} \cdots \mathrm{N}$ hydrogen bonds and three $\mathrm{I}^{-} \ldots \mathrm{Li}$ ionic bonds (Figure 1e). Examples of these cations show that we can actually steer the coordination number from tri- (Figure 1c), tetra- (Figure 1d)
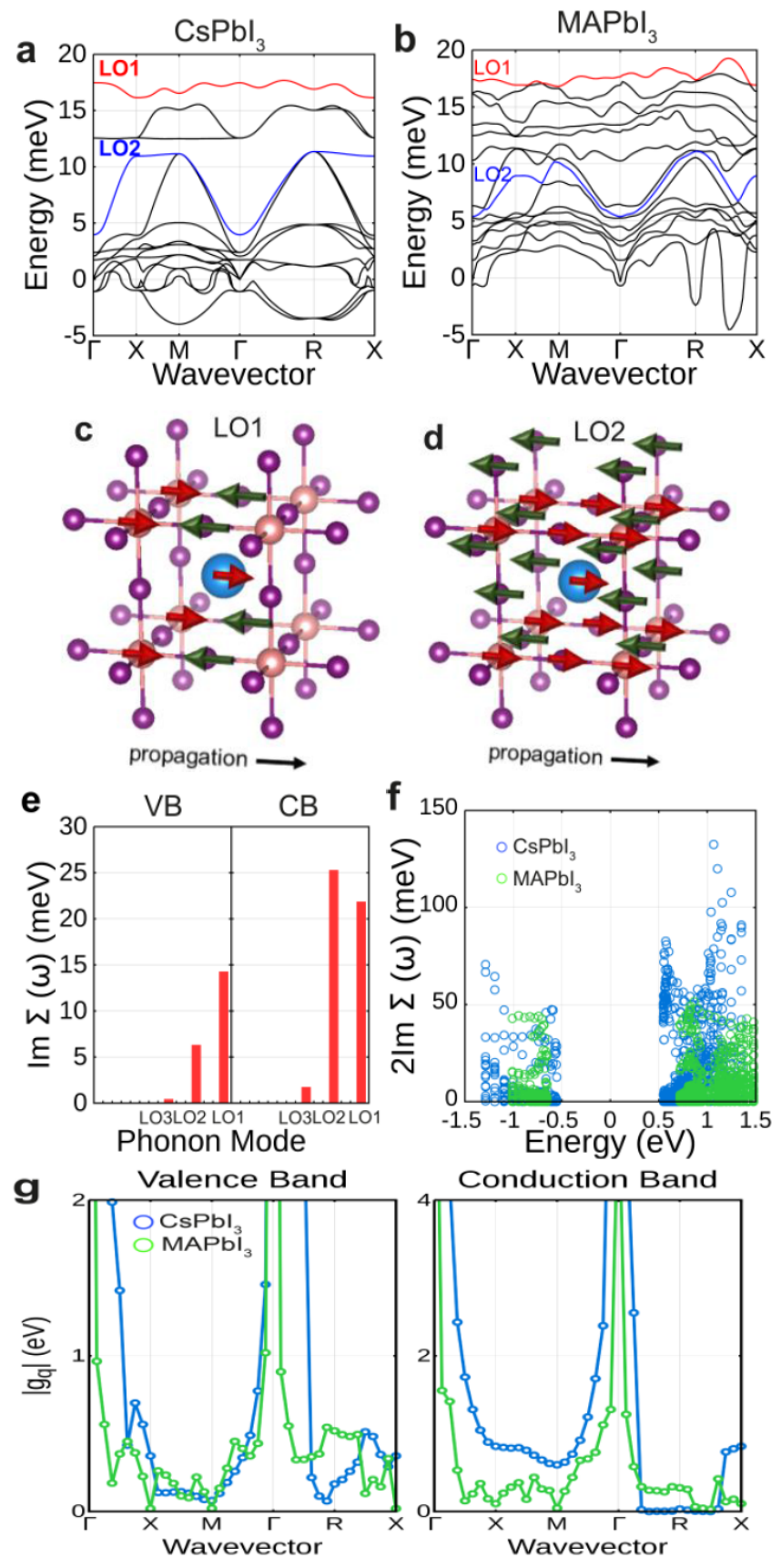

FIG. 2. a) $\mathrm{CsPbI}_{3}$ and b) $\mathrm{MAPbI}_{3}$ with $\mathrm{LO} 1$ (red) and $\mathrm{LO} 2$ (blue) that mostly contribute to e-ph coupling in VB and CB. The eigenmode of c) LO1 and d) LO2 in $\mathrm{CsPbI}_{3}$. e) Electronphonon self-energy of $\mathrm{VB}$ and $\mathrm{CB}$ at $\mathrm{R}(0.5,0.5,0.5)$ of $\mathrm{BZ}$ with three dominant LO modes: LO1, LO2, and LO3 (3rd highest LO mode). f) Scattering rate $\tau^{-1}$ or $2 \operatorname{Im}\{\Sigma(k, \omega)\}$ for $\mathrm{CsPbI}_{3}$ (blue) and $\mathrm{MAPbI}_{3}$ (green) along $(\eta, \eta, \eta)$ to $\mathrm{R}(0.5$, $0.5,0.5)$ and $\mathrm{R}(0.5,0.5,0.5)$ to $(0.5, \eta, 0.5)$ with $0.45 \geq \eta \geq$ 0.5 at $330 \mathrm{~K} . E_{F}$ is set to $0 \mathrm{eV}$. g) Frhlich vertex $(m=n)$ of $\mathrm{CsPbI}_{3}$ (blue) and $\mathrm{MAPbI}_{3}$ (green) for VB (left) and $\mathrm{CB}$ (right) with the typical divergence of polar materials for long range interactions. 
to penta-coordination (Figure 1e) with still very strong bonding strength by employing different organic cations.

Since A-site cations are not located in the low energy spectrum of electronic structure at all, they are hardly expected to participate in carrier transport. However, it turns out that the choice of A-site cation is crucial because of polaronic nature of PSCs. It is clear from the examples of two well-known materials: cubic $\mathrm{CsPbI}_{3}$ and $\mathrm{MAPbI}_{3}$. Phonon dispersions (Figure 2a, 2b) show a large LO(longitudinal optical)-TO(transverse optical) splitting due to the large Born effective charges of $\mathrm{Pb}$ and $\mathrm{I}$. Long range macroscopic Coulomb interaction in non-analytic dynamical matrix of polar crystal at the $\Gamma$ point introduces the LO-TO splitting, described by the Lynddane-Sachs-Teller (LST) relation. 34 Although LST does not hold exactly in polyatomic case such as PSCs, this relation can be a measure of crystal polarizability and we tabulated it in Table 1 as a reference. Organic cations including $\mathrm{MA}^{+}$diminish the splitting (Table 1), indicating that they effectively lower long range Coulomb interactions. Compared to $\mathrm{CsPbI}_{3}(17.47 \mathrm{meV})$, the energy $\left(E_{L O 1}\right)$ of the highest $\mathrm{LO}$ mode (LO1) decreases in the case of $\mathrm{MAPbI}_{3}(16.21$ meV). In $\mathrm{CsPbI}_{3}$, the clear separation between heavy $\mathrm{Cs}^{+}$modes (which mostly have low energies $5 \mathrm{meV}$ ) and $\mathrm{PbI}_{3}^{-}$optical phonon modes (i. $5 \mathrm{meV}$ ) is noted (Figure $2 \mathrm{a}$ ). In $\mathrm{MAPbI}_{3}$, the rotation/translation modes of $\mathrm{MA}+$ are mixed with the $\mathrm{PbI}_{3}^{-}$LO modes (Figure 2b). A light molecule such as $\mathrm{MA}^{+}$which interacts with $\mathrm{I}^{-}$ by anisotropic directional hydrogen bonding breaks the symmetry of $\mathrm{PbI}_{3}$ lattice, and effectively suppresses the $\mathrm{PbI}_{3}^{-} \mathrm{LO}$ lattice vibrations by the linear $\mathrm{I}^{-} \ldots \mathrm{HN}$ halogenhydrogen bonding perpendicular to the $\mathrm{Pb}^{2+}-\mathrm{I}^{-}-\mathrm{Pb}^{2+}$ perovskite skeleton.

A calculation of e-ph coupling for all modes reveals that most contributions of e-ph scattering in both $\mathrm{CsPbI}_{3}$ and $\mathrm{MAPbI}_{3}$ at band edges of valence band (VB) and conduction band (CB) come from LO modes: the highest LO mode LO1 (red) and the second highest LO mode LO2 (blue) (Figure 2a, 2b). Except LO1 (Figure 2c) and LO2 (Figure 2d), other 13 modes' contributions are almost negligible or completely insignificant (Figure 2e). Therefore, by observing these two dominant modes, it would be easy to track the changes in e-ph coupling, once other cations are substituted for the $\mathrm{MA}^{+}$in the A-site. In $\mathrm{CsPbI}_{3}$, due to large polarizability of the $\mathrm{Pb}^{2+}$ $\mathrm{I}^{-}$bond and large proportion of $\mathrm{PbI}_{3}^{-} \mathrm{LO}$ vibration, the e-ph scattering is much larger than in $\mathrm{MAPbI}_{3}$ (Figure 2f). As the inorganic cation $\mathrm{Cs}^{+}$is replaced by $\mathrm{MA}^{+}$, the e-ph coupling diminishes because of the suppression of $\mathrm{PbI}_{3}^{-} \mathrm{LO}$ phonon modes by $\mathrm{MA}^{+}$which is attached to $\mathrm{I}^{-}$atoms. The result agrees with our design principle that organic cations like $\mathrm{MA}^{+}$should decrease the scattering rate compared to inorganic PSCs (Figure 2f). The Frhlich vertex $(m=n)$ of $\mathrm{CsPbI}_{3}$ (blue) and $\mathrm{MAPbI}_{3}$ (green) for LO1 and LO2 indicates that in addition to the overall reduced e-ph couplings, long range interac- tions $(q \rightarrow 0)$ are substantially quenched for $\mathrm{MAPbI}_{3}$ (Figure 2g). These overall observations point out that properly chosen organic cations behave much better in charge mobility than inorganic ones.

Negative (imaginary) acoustic modes, for example at $\mathrm{M}$ (tetragonal) and $\mathrm{R}$ (orthorhombic) in $\mathrm{CsPbI}_{3}$, indicate instability of cubic structure at $0 \mathrm{~K}$. However, because we are solely interested in the optical modes, particularly LO, our calculation can be justified if LO spectra do not change much at high temperatures. Fourier transformed velocity auto-correlation function (VAC) from ab initio molecular dynamics (MD) simualtions at $600 \mathrm{~K}$ above the critical temperature $T_{C}(583 \mathrm{~K}) 35$ for the cubic phase (Figure S1a, Supporting Information) shows that the overall MD spectra agree with $0 \mathrm{~K}$ lattice phonon density of states (DOS), and both LO1 and LO2 energy DOS do not change much (Figure S1b, Supporting Information). Therefore, we assume that at high temperatures, phonon spectra of high energy LO modes are not severely affected.

Among the tested A-site cations, we have chosen the most promising cations: $\mathrm{HA}^{+}, \mathrm{fA}^{+}, \mathrm{LM}^{+}$, and $\mathrm{TA}^{+}$according to the coordination number $\left[\mathrm{MA}^{+}(3)\right.$ $\left.<\mathrm{HA}^{+}(4)<\mathrm{LM}^{+}(5)\right]$ and the coordinating distances $\left[\mathrm{d}\left(\mathrm{fA}^{+}\right), \mathrm{d}\left(\mathrm{TA}^{+}\right), \mathrm{d}\left(\mathrm{HA}^{+}\right)<\mathrm{d}\left(\mathrm{MA}^{+}\right)\right]$. The Born effective charges of I atoms coordinated by those organic cations decrease as $Z_{\mathrm{MAPbI}_{3}}^{*}>Z_{\mathrm{LMPbI}_{3}}^{*}>Z_{\mathrm{HAPbI}_{3}}^{*}>$ $Z_{f \mathrm{AbI}_{3}}^{*}>Z_{\mathrm{TAPbI}}^{*}$ (Table 1). Being substituted by $\mathrm{O}$, $\mathrm{F}$, or being fluorinated, the cations working as a buffer are able to reduce the effective $\mathrm{Pb}-\mathrm{I}$ dipole. The coordination of cations can reduce $Z^{*}$ up to $40 \%$ compared to non-coordinated one in the case of fAPbI3 (table $\mathrm{S} 1$, Supporting Information).

The coordination number is also important since $\mathrm{Pb}^{2+}$. $\mathrm{I}^{-}$LO eigenmodes are absorbed by organic cations via coordination. (Figure 3) The increase in coordination number decreases the value of , for example, $\mathrm{HA}^{+}(1.26)$ (Figure 3b) and $\mathrm{LM}^{+}(1.33)$ (Figure $3 \mathrm{~d}$ ) compared to $\mathrm{MA}^{+}(1.46)$ (Figure $2 \mathrm{~b}$ and Table 1). $\mathrm{LMPbI}_{3}$ shows a helpful example of how the $\mathrm{Pb}-\mathrm{I}$ motion loses its contribution in LO1 and LO2 as the number of organic cations coordinated to I increases. As for the $\mathrm{LM}^{+}$ cation (Figure 3e), the degree of mixing and screening of LO modes by the cation is maximized among the candidate cations by halogen-hydrogen bonding and directional linear $\mathrm{I}^{-} \ldots \mathrm{LiNH}_{2}$ halogen-lithium bonding, followed by $\mathrm{HA}^{+}$. The $\mathrm{HA}^{+}$cation being tetra-coordinated to I also absorbs the $\mathrm{Pb}-\mathrm{I} \mathrm{LO}$ mode more significantly than the $\mathrm{MA}^{+}$cation. (Figure $3 \mathrm{e}$ )

The overall discussions can be summed up in Figure 4. It turns out that, in $\mathrm{VB}$ and $\mathrm{CB}, \mathrm{TA}^{+}$which was once conjectured as a promising cation is not a good candidate for carrier transport, because a new LO motion between $\mathrm{F}$ and $\mathrm{Pb}-\mathrm{I}$ is present in $\mathrm{TAPbI}_{3}$; this additional LO mode gives rise to the largest e-ph scattering (Figure $\mathrm{S} 2$, Supporting Information). In $\mathrm{VB}$, the scattering rates of $\mathrm{HA}^{+}$and $\mathrm{LM}^{+}$are affixed to the value of $\mathrm{MA}^{+}$. 

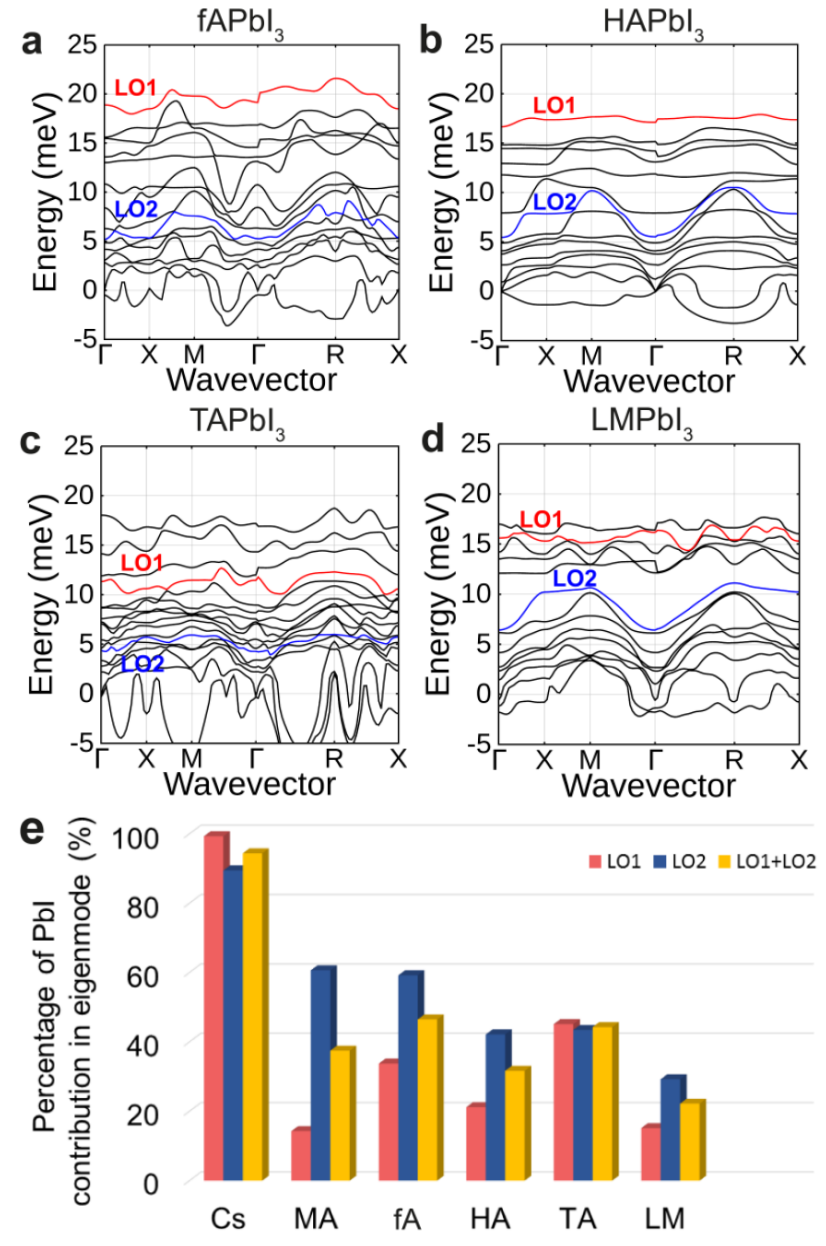

FIG. 3. Vibrational dispersions of a) $\mathrm{fAPbI}_{3}$, b) $\mathrm{HAPbI}_{3}$, c) $\mathrm{TAPbI}_{3}$, d) $\mathrm{LMPbI}_{3}$. e) Histogram of dynamical matrix components of LO eigenmodes for LO1 (red), LO2 (blue), and its average (yellow). A small proportion of A-site cation component in LO modes in CsPbI3 substantially increases by modes of the organic cations. Eigenmode contributions of Asite cation in LO modes are $\mathrm{Cs}^{+}(5.6 \%), \mathrm{MA}^{+}(62.5 \%), \mathrm{fA}^{+}$ $(53.5 \%), \mathrm{HA}^{+}(68.3 \%), \mathrm{TA}^{+}(55.7 \%)$, and $\mathrm{LM}^{+}(77.8 \%)$. The eigenmode components of $\mathrm{Pb}-\mathrm{I}$ are absorbed by A-site organic cations.

However, it is interesting to note that the $\mathrm{fA}^{+}$cation, which buffers a large polarizability between $\mathrm{Pb}$ and $\mathrm{I}$ (Table 1), reduces the scattering rate to half the value of $\mathrm{MA}^{+}$(Figure 4a). In $\mathrm{CB}$, a reduction of the scattering rate is clear for $\mathrm{fA}^{+}, \mathrm{HA}^{+}$, and $\mathrm{LM}^{+}$. In particular, $\mathrm{LM}^{+}$significantly reduces the e-ph scattering (Figure 4b) because of its largest mixing with $\mathrm{Pb}-\mathrm{I}$ LO modes (Figure 3e). Electron self-energy of Rayleigh-Schrodinger perturbation theory for nondegenerate conduction band minimum at $\Gamma$ of Brillouin Zone (BZ) is simply given by $\operatorname{Re}\{\Sigma(k, \omega)\}=-\alpha \hbar \omega_{L O}$ at $0 \mathrm{~K}$, where $\alpha$ is polaron coupling constant, $\alpha=\frac{e^{2}}{8 \pi \epsilon_{0} \hbar \omega_{L O}}\left(\frac{2 \omega_{L O} m_{0}}{\hbar}\right)^{1 / 2}\left(\frac{1}{\epsilon_{\infty}}-\frac{1}{\epsilon_{0}}\right) \cdot[36$. Although this relation is from a simple model Hamiltonian, still can provide a good measure of polaron coupling for real materials such as PSCs. Thus, the comparison of polaron coupling constants at $0 \mathrm{~K}$ $\left[\alpha\left(\mathrm{CsPbI}_{3}\right)=1.42, \alpha\left(\mathrm{MAPbI}_{3}\right)=0.11, \alpha\left(\mathrm{HAPbI}_{3}\right)\right.$ $=0.036$, and $\alpha\left(\mathrm{LMPbI}_{3}\right)=0.026$ (Figure 4c)] reveals that the prospective organic cations reduce the polaron coupling effectively. As confirmed by other DFT calculations, 37] we note that strong hydrogen bonding of cations introduces a change of simple quadratic band minimum (or maximum) into a double well like shape (Figure 4c). Photo-luminescence (PL) bandwidths as the function of temperatures (Figure 4d) confirm that $\mathrm{HA}^{+}$and $\mathrm{fA}^{+}$with the strong coordination to I and $\mathrm{LM}^{+}$with a large coordination number to $\mathrm{I}$ are promising A-site organic cations for highly efficient PSCs. Finally, from a concise relation for the mobility $\mu=e \tau / m^{*}\left(\mathrm{~cm}^{2} V^{-1} s^{-1}\right)$, where $e$ is carrier charge, $\tau$ is the calculated lifetime of PSCs, and $m^{*}$ the effective mass of $\mathrm{CB}$ and $\mathrm{VB}, 38$ we estimate the mobility for the prospective materials at $330 \mathrm{~K}$ for electrons, $\mu_{\mathrm{TAPbI}_{3}}(61.85)<\mu_{\mathrm{CsPbI}_{3}}(73.94)<\mu_{\mathrm{MAPbI}_{3}}(118.08)<$ $\mu_{\mathrm{HAPbI}_{3}}(150.82)<\mu_{\mathrm{fAPbI}_{3}}(162.92)<\mu_{\mathrm{LMPbI}_{3}}(464.41)$, and for holes $\mu_{\mathrm{TAPbI}_{3}}(57.88)<\mu_{\mathrm{CsPbI}_{3}}(98.11)<$ $\mu_{\mathrm{HAPbI}_{3}}(102.22)<\mu_{\mathrm{MAPbI}_{3}}(108.70)<$ $\mu_{\mathrm{LMPbI}_{3}}(110.25)<\mu_{\mathrm{fAPbI}_{3}}(226.77)$.

A-site cations have not been considered as a main factor for carrier transport but been neglected except its role in structural tolerance for cubic perovskite. Here, we shed a light on the hidden role of A-site cation in PSCs within Frhlich polaron picture. From this perspective, we found a design principle of choosing a suitable cation for a better carrier mobility to boost efficiency of PSCs. The key for steering the charge transport is the coordination of organic cations to I atoms. By increasing the interaction strength of the A-site cation to I and the number of organic cations coordinated to I, the vibrational mode of molecular cations involved in directional $\mathrm{I}-\mathrm{HN}(\mathrm{O})$ halogen-hydrogen or $\mathrm{I}^{-} \ldots \mathrm{Li}$ bonding weakens e-ph interaction because of the reduction in $Z^{*}(P b, I)$ and eigenmode components of $\mathrm{Pb}$ and $\mathrm{I}$. We have calculated polaron properties of lead iodide PSCs with various types of A-site cations from first principles. As compared with the most-used $\mathrm{MA}^{+}$organic cation and inorganic cation $\mathrm{Cs}^{+}$, the untapped organic cation $\mathrm{HA}^{+}$ and other cations similar to $\mathrm{LM}^{+}$are likely to be highly promising for light harvesting. Because most of hole conducting materials (HTM) contain $\mathrm{Li}^{+}$ions in lithiumbis(trifluoromethylsulphonyl)imide (LiTFSI), the formation of $\mathrm{LM}^{+}$at the interface of HTM could be possible by diffusion of $\mathrm{Li}$ ions followed by heating in consideration that $\mathrm{LM}^{+}$is not necessary to be one molecular cation but a molecular system of $\mathrm{Li}^{+} \ldots \mathrm{NH}_{2} \mathrm{CH}_{3}$ where a $\mathrm{Li}^{+}$cation is solvated by $\mathrm{NH}_{2} \mathrm{CH}_{3}$ and three $\mathrm{I}^{-}$anions. This is quite plausible in the presence of three $\mathrm{I}^{-}$anions because $\mathrm{Li}^{+}$ is known to be solvated by $\mathrm{NH}_{2} \mathrm{CH}_{3}, 39$, 40, and hence it is considered as a possible process to provide such a system. To conclude, we have uncovered a new perspective on the role of the A-site organic cation that reduces the 

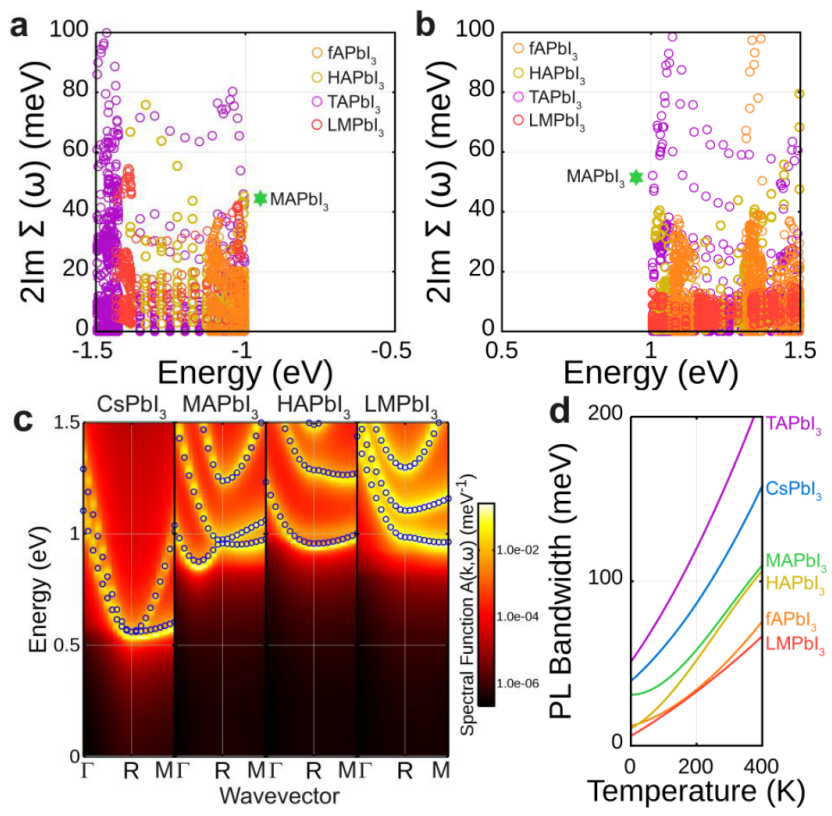

FIG. 4. a) Hole and b) electron scattering rate $\tau^{-1}$ or $2 \operatorname{Im}\{\Sigma(k, \omega)\}$ for $\mathrm{fAPbI}_{3}$ (orange), $\mathrm{HAPbI}_{3}$ (yellow), $\mathrm{TAPbI}_{3}$ (purple), $\mathrm{LMPbI}_{3}$ (red) at band minimum or maximum along $\mathrm{k}=(\eta, \eta, \eta)$ to $\mathrm{R}(0.5,0.5,0.5)$ and $\mathrm{R}(0.5$, $0.5,0.5)$ to $(0.5, \eta, 0.5)$ with $0.45 \geq \eta \geq 0.5$ at $330 \mathrm{~K}$. Band maxima(minima) are aligned so that it can be easily recognized. of $2 \operatorname{Im}\{\Sigma(k, \omega)\} \mathrm{MAPbI}_{3}$ at band edges are indicated as star (green). c) Electron spectral function of $\mathrm{CsPbI}_{3}, \mathrm{MAPbI}_{3}, \mathrm{HAPbI}_{3}$, and $\mathrm{LMPbI}_{3}$ along with DFT Kohn-Sham eigenvalues (blue circles) in the vicinity $\mathrm{R}(0.5,0.5,0.5)$ of $\mathrm{BZ}$ at $0 \mathrm{~K}$. Real part of electron selfenergy $\operatorname{Re}\{\Sigma(k, \omega)\}=\alpha \hbar \omega_{L O}$ indicates the strength of eph coupling for various A-site organic cations. d) Photoluminescence (PL) bandwidth as the function of temperatures for $\mathrm{TAPbI}_{3}$ (purple), $\mathrm{CsPbI}_{3}$ (blue), $\mathrm{MAPbI}$ (green), $\mathrm{HAPbI}_{3}$ (yellow), fAPbI 3 (orange), and $\mathrm{LMPbI}_{3}$ (red).

Frohlich e-ph coupling of the $\mathrm{PbI}_{3}^{-} \mathrm{LO}$ vibration. The proposed design principle could help find a new prospect of A-site organic cation for the development of PSCs to expedite effective light harvesting.

We calculated ionic radius of organic cations using PBE functional and aug-cc-pVTZ basis set. Quantum ESPRESSO package v.6.1 41] were used for DFT, DFPT, and ab initio MD. We used norm-conserving PBE pseudopotential including $\mathrm{d}$ core states of $\mathrm{Pb}$ and sampled an unshifted $(8 \times 8 \times 8)$ grid in BZ for geometry optimization and for electronic calculation at energy cutoff of 80 Ry for PSCs containing organic cations and 100 Ry for $\mathrm{CsPbI}_{3}$. Because lattice distortion is not significantly affected by types of functional and spin-orbit coupling, 42. we used PBE exchange functional. We used DFPT for phonon dispersions and considered the non-analytic term of dynamical matrix at $\Gamma$ of BZ to account for LO-TO splitting. The e-ph coupling and electron self-energy were calculated using EPW v.4.1.0 [4] with unshifted $(64 \times 64 \times 64)$ q-points and with Gaussian broadening
$10 \mathrm{meV}$ for BZ integration. We performed ab initio MD simulations with time step $\mathrm{t}=0.50 \mathrm{fs}$ and total duration of $10 \mathrm{ps}$ at $600 \mathrm{~K}$. Fourier transformed velocity autocorrelation(VAC) was done for 9 ps excluding 1 ps of initial thermalization and was smoothened by SavitzkyGolay filter.

This work was supported by National Honor Scientist Program (2010-0020414) of NRF. Computation was supported by KISTI (KSC-2016-C3-0074). C.W.M. performed all calculations. J. H. Y. calculated VAC spectrum of ab initio MD. All authors analyzed the data and wrote the manuscript.

[1] W. S. Yang, B.-W. Park, E. H. Jung, N. J. Jeon, Y. C. Kim, D. U. Lee, S. S. Shin, J. Seo, E. K. Kim, J. H. Noh, and S. I. Seok, Science 356, 167 (2017).

[2] Y. Yang, D. P. Ostrowski, R. M. France, K. Zhu, J. v. Lagemaat, J. M. Luther, and M. C. Beard, Nat. Photon. 10, 53 (2016).

[3] J. Even, L. Pedesseau, and C. Katan, J. Phys. Chem. C 118, 11566 (2014).

[4] J. S. Manser and P. V. Kamat, Nat. Photon. 8, 737 (2014).

[5] N. Park, M. Gratzel, T. Miyasaka, K. Zhu, and K. Emery, Nat. Energy 1, 16152 (2016).

[6] C. Wehrenfennig, G. E. Eperon, M. B. Johnston, H. J. Snaith, and L. M. Herz, Adv. Mater. 26, 1584 (2014).

[7] C. C. Stoumpos, C. D. Malliakas, and M. G. Kanatzidis, Inorg. Chem. 52, 9019 (2013).

[8] C. Wehrenfennig, M. Liu, H. J. Snaith, M. B. Johnston, and L. M. Herz, Energy Environ. Sci. 7, 2269 (2014).

[9] J. M. Azpiroz, E. Mosconi, J. Bisquert, and F. DAngelis, Energy Environ. Sci. 8, 2118 (2015).

[10] C. Wehrenfennig, M. Liu, H. J. Snaith, M. B. Johnston, and L. M. Herz, J. Phys. Chem. Lett. 5, 1300 (2014).

[11] R. L. Milot, G. E. Eperon, H. J. Snaith, M. B. Johnston, and L. M. Herz, Adv. Mater. 25, 62186227 (2015).

[12] D. A. Valverde-Chavez, C. S. Ponseca, C. C. S. , A. Yartsev, M. G. Kanatzidis, V. Sundstram, and D. G. Cooke, Energy Environ. Sci. 8, 3700 (2015).

[13] C. Sheng, C. Zhang, Y. Zhai, K. Mielczarek, W. Wang, W. Ma, A. Zakhidov, and Z. V. Vardeny, Phys. Rev. Lett. 114, 116601 (2015).

[14] A. M. A. Leguy, J. M. Frost, A. P. McMahon, V. G. Sakai, W. Kockelmann, C. Law, X. Li, F. Foglia, A. Walsh, B. C. ORegan, J. Nelson, J. T. Cabral, and P. R. F. Barnes, Nature Commun. 6, 7124 (2015).

[15] C. Eames, J. M. Frost, P. R. F. Barnes, B. C. ORegan, A. Walsh, and M. S. Islam, Nature Commun. 6, 7497 (2015).

[16] A. A. Zhumekenov, M. I. Saidaminov, M. A. Haque, E. Alarousu, B. M. S. P. Sarmah, I. Dursun, X. Miao, A. L. Abdelhady, T. Wu, O. F. Mohammed, and O. M. Bakr, ACS Energy Lett. 1, 32 (2016).

[17] H. Oga, A. Saeki, Y. Ogomi, S. Hayase, and S. Seki, J. Am. Chem. Soc. 136, 1381813825 (2014).

[18] Y. He and G. Galli, Chem. Mater. 26, 53945400 (2014).

[19] A. D. Wright, C. Verdi, R. L. Milot, G. E. Eperon, M. A. Perez-Osorio, H. J. Snaith, F. Giustino, M. B. Johnston, and L. M. Herz, Nature Commun. 7, 11755 (2016). 
[20] K. Wu, A. Bera, C. Ma, Y. Du, Y. Yang, L. Lib, and T. Wu, Phys. Chem. Chem. Phys. 16, 22476 (2014).

[21] M. T. Weller, O. J. Weber, P. F. Henry, A. M. D. Pumpo, and T. C. Hansen, Chem. Commun. 51, 4180 (2015).

[22] J. Feng, APL Materials 2, 081801 (2014).

[23] H. Frohlich, Adv. Phys. 3, 325361 (1954).

[24] C. Verdi and F. Giustino, Phys. Rev. Lett. 115, 176401 (2015).

[25] V. M. Goldschmidt, Naturwissenschaften 14, 477485 (1926).

[26] G. Kieslich, S. Sun, and A. K. Cheetham, Chem. Sci. 5, 4712 (2014).

[27] M. R. Filip, G. E. Eperon, H. J. Snaith, and F. Giustino, Nat. Commun. 5, 6757 (2014).

[28] L. Xie, L. Chen, Z. Nan, H. Lin, T. Wang, D. Zhan, J. Yan, B. Mao, and Z. Tian, J. Am. Chem. Soc. 139, 33203323 (2017).

[29] F. Giustino, Rev. Mod. Phys. 89, 015003 (2017).

[30] B. Jerslev, Nature 160, 641 (1947).

[31] V. Grakauskas, A. H. Remanick, and K. Baum, J. Am. Chem. Soc. 90, 3839 (1968).

[32] U. A. Jayasooriya, C. J. Ludman, C. I. Ratcliff, and T. C. Waddington, J. Chem. SOC., Faraday Trans. 2 77, 287 (1981).
[33] T. E. Salter and A. M. Ellis, J. Chem. Phys. 127, 144314 (2007).

[34] R. H. Lyddane, R. G. Sachs, and E. Teller, Phys. Rev. 59, 673 (1941).

[35] J. B. Hoffman, A. L. Schleper, and P. V. Kamat, J. Am. Chem. Soc. 138, 8603 (2016).

[36] O. Madelung, Introduction to solid-state theory (Springer-Verlag: Berlin Heidelberg).

[37] C. Motta, F. El-Mellouhi, S. Kais, N. Tabet, F. Alharbi, and S. Sanvito, Nature Commun. 6, 7026 (2016).

[38] P. Umari, E. Mosconi, and F. D. Angelis, Sci. Rep. 4, 4467 (2014).

[39] M. K. Faber, O. FussB-Rydel, J. B. Skowyra, L. E. H. McMills, and J. L. Dye, J. Am. Chem. Soc. 111, 5957 (1989).

[40] S. Hayama, J. C. Wasse, N. T. Skipper, and J. K. Walters, Mol. Phys. 779, 035603 (2001).

[41] P. Giannozzi et al., J.Phys.: Condens. Matter 21, 395502 (2009).

[42] A. J. Neukirch, W. Nie, J. Blancon, K. Appavoo, H. Tsai, M. Y. Sfeir, C. Katan, L. Pedesseau, J. Even, J. J. Crochet, G. Gupta, A. D. Mohite, and S. Tretiak, Nano Lett. 16, 3809 (2016).

[43] S. Ponce, E. R. Margine, C. Verdi, and F. Giustino, Comput. Phys. Commun. 209, 116 (2016). 